\title{
TEXTURE AND STRENGTH EVOLUTION IN DEFORMED POLYPROPYLENE
}

\author{
J. R. HIRSCH and P. T. WANG \\ ALCOA Center, Pittsburgh, PA 15069, USA
}

\begin{abstract}
Semicrystalline polypropylene samples with high concentrations of crystallinity ( $\gtrsim 62 \%)$ are deformed in uniaxial compression at different temperatures and different strain rates. The orientation changes of the crystalline part of the material are analyzed using X-ray diffraction methods. Pole figure evaluation is used to obtain information about the crystal structure, the geometry of slip, and its correlation with the characteristic of strength evolution in polymers. The contribution of the crystalline part of the material to plastic flow is estimated and the textures are related to the characteristic effects in plastic and visco-elastic response of the material during deformation and subsequent stress relaxation. The observed texture effects can be explained by the rotation of crystallographic $c$-axis (backbone axis) normal to the compression direction at high temperatures in a planar slip mode. The stress/strain and relaxation behavior of the material is interpreted as a combination of viscoelastic flow of amorphous volume and irreversible plastic flow of the crystalline part which contributes to an orientation dependence of deformation and stress relaxation.
\end{abstract}

KEY WORDS Polypropylene, uniaxial compression, X-ray diffraction, pole figures, stress-strain behavior, stress relaxation.

\section{INTRODUCTION}

Solid state sheet processing for semicrystalline polymers has gained significant interest in recent years due to its capability of enhancing material properties by imposing an uniaxial or biaxial deformation on polymeric chains. In order to avoid the planar anisotropy in sheet which can cause the instability at the subsequent forming process (DiPede and Woodhams, 1989), the biaxial deformation process becomes more attractive than the uniaxial one. Recent process development has concluded that a twin belt process (Lo and Humphries, 1989) is of major benefit in which the biaxially oriented sheet is produced continuously with uniform material properties throughout the thickness, and superior to those obtained in the hydrostatic extrusion process (Austen and Humphries, 1981; Hiltner et al., 1987).

In general, the degree of molecular orientation can be inferred from the improvements of tensile modulus and strength, and flexural modulus, or even from the flow stress measurement as a function of stretch ratio along the deformation direction on a macroscale, which is also called orientation ratio. However, in a more detailed thermomechanical deformation process where strain, strain rate and temperature are controlled, the macroscale representation is not sensitive enough to describe the evolution of molecular orientation with processing. This is done particularly for semi-crystalline material where two phases, crystalline and amorphous, are interacting with each other during deformation. Hence, microscale orientation measurements using wide and small angle X-rays (Katt and Schultz, 1982; Barnes and Clark, 1986), infrared 
spectroscopy (Samuels, 1974), or polarized microwaves (Osaki, 1987) are necessary to quantify the microstructural changes with deformation if a realistic constitutive model is to be developed.

To date, none of the orientation data available in literature has considered the strain rate effect during deformation. In this paper, the texture and strength evolution of biaxially deformed polypropylene (PP) are investigated at various thermomechanical deformation histories. Semicrystalline polypropylene samples with relatively high fraction of crystallinity $(~ ₹ 62 \%)$ are deformed in uniaxial stress compression at two temperature and strain rate levels. The tests are conducted under prescribed strain, strain rate and temperature conditions so process conditions are simulated and the evolution of texture and strength can be evaluated for varying process parameters. The orientation changes in the crystalline phase of polypropylene are analyzed using X-ray diffraction methods. With the understanding of crystalline behavior during orientation process, the deformation energy for the amorphous phase can be extrapolated and discussed separately. Thus, an advanced constitutive model for semicrystalline material can be proposed.

\section{EXPERIMENTAL PROCEDURES AND RESULTS}

\section{Materials and Test Parameters}

Two different types of materials were used:

I) Medium Molecular Weight Polypropylene (MMW-PP) (Himont 6823) and

II) Ultra High Molecular Weight (UHMW-PP) Polypropylene (Aristech).

Cylindrical samples ( 1 in. diameter and 0.5 in. height) were deformed under uniaxial stress compression at isothermal and true strain rate conditions using a servo-hydraulic controlled tester. All samples underwent monotonic compressive deformation at various strain rates $(0.01 / \mathrm{sec}$ to $10 / \mathrm{sec})$ to a specific strain level and then were quenched under load to freeze the structure as well as to minimize the distortion of the flat, disc-like shape. Samples were deformed to $180 \%$ strain (which is about 0.083 in. in final thickness); some UHMW-PP samples were also deformed to $60 \%$ and $120 \%$ strain to study the evolution of texture with deformation. Some reference samples were heated to the testing temperature and immediately quenched without deformation. Stress relaxation measurements were conducted right after compression to $180 \%$ true strain for some of the samples. The details of experimental procedure can be referred to Wang et al. (1989). The data from these tests were analyzed to show the stability of polypropylene structure being deformed and to rationalize the contribution between amorphous and crystalline parts qualitatively.

The following abbreviations are used to define the different deformation conditions:

00 heated and quenched (undeformed $=$ reference sample)

Material was deformed at two temperatures:

$$
\begin{aligned}
\mathrm{LT} & =\text { low Temperature }\left(135^{\circ} \mathrm{C}\right) \\
\mathrm{HT} & =\text { high Temperature }\left(155^{\circ} \mathrm{C}\right)
\end{aligned}
$$


and at two strain rates:

LS low strain rate $(\dot{\varepsilon}=0.1 / \mathrm{sec})$

HS high strain rate $(\dot{\varepsilon}=1 / \mathrm{sec}$ or $10 / \mathrm{sec})$

(The higher strain rate of $\dot{\varepsilon}=10 / \mathrm{sec}$ provided no suitable samples for texture measurements in UHMW-PP.)

\section{Results of Texture Measurements}

Figures 1 and 2 show the reflected $\mathrm{X}$-ray intensity plotted versus the Bragg angle $\theta$ for UHMW-PP as an example. These $\theta$ scan plots show the formation of peaks at different $\theta$ angles due to specific crystallographic planes. The first two Bragg-reflexes (110) and (040) were selected for pole figure measurements. The strongest texture effect occurs for low strain rate deformation (LS) and at high temperatures (HT) (Figures 1 and 2b). At low temperature (Figure 2a) the peaks are much less pronounced, or even seem somewhat decreased compared to those of the reference sample (00). For the deformed material, generally a somewhat higher background level is observed which indicates some loss of crystalline volume. A significant peak shift is most pronounced for low temperature deformation. In Table 1a, the exact Bragg angles of the peak maxima and mean

UHMW polypropylene - Theto scan

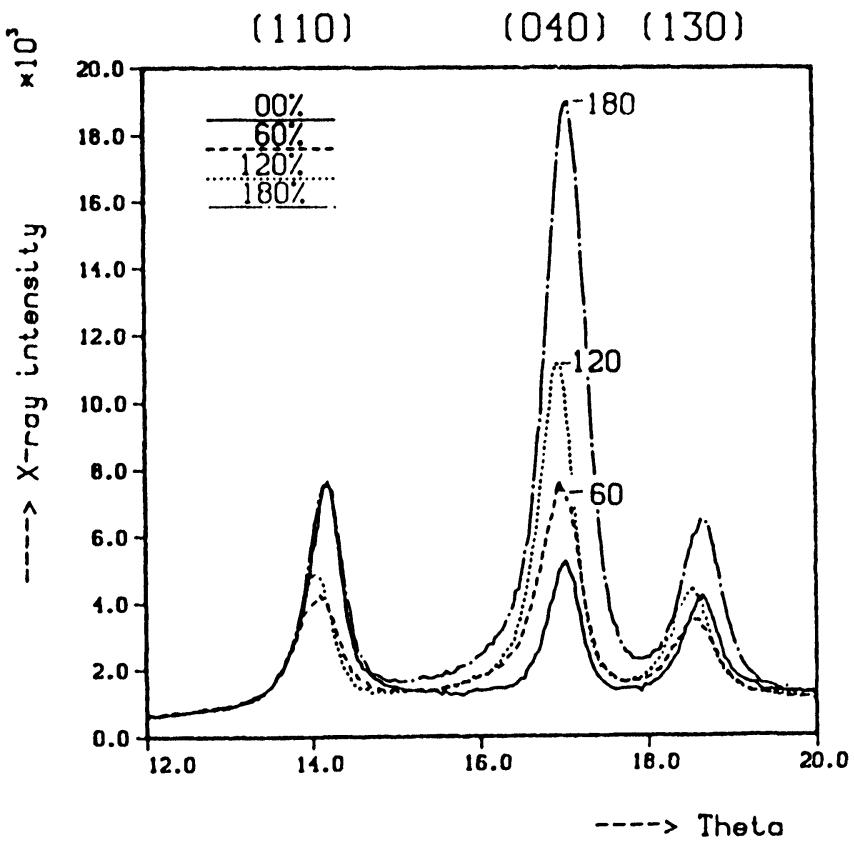

Figure 1 Reflected X-ray intensity of UHMW-polypropylene versus reflection angle $\theta$ ( $\theta$ scan) deformed to different strains at low strain rate $(0.01 / \mathrm{sec})$. 

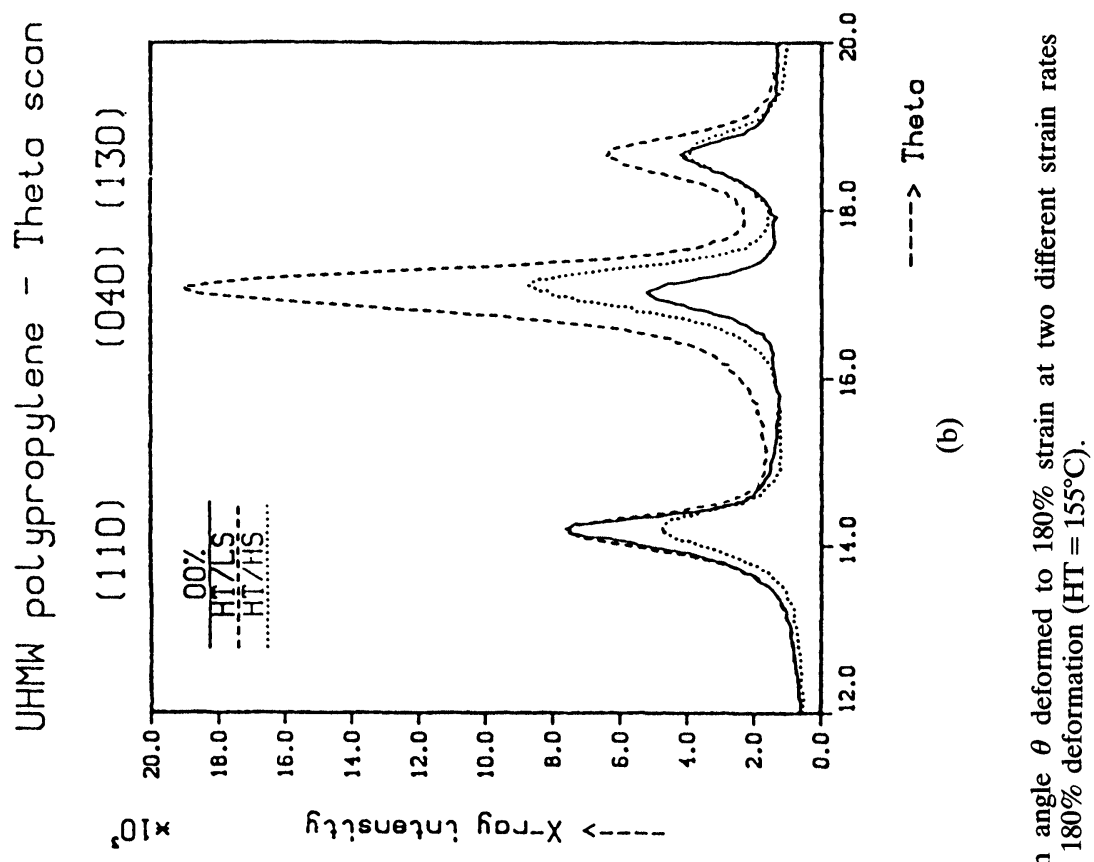

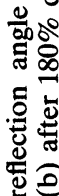

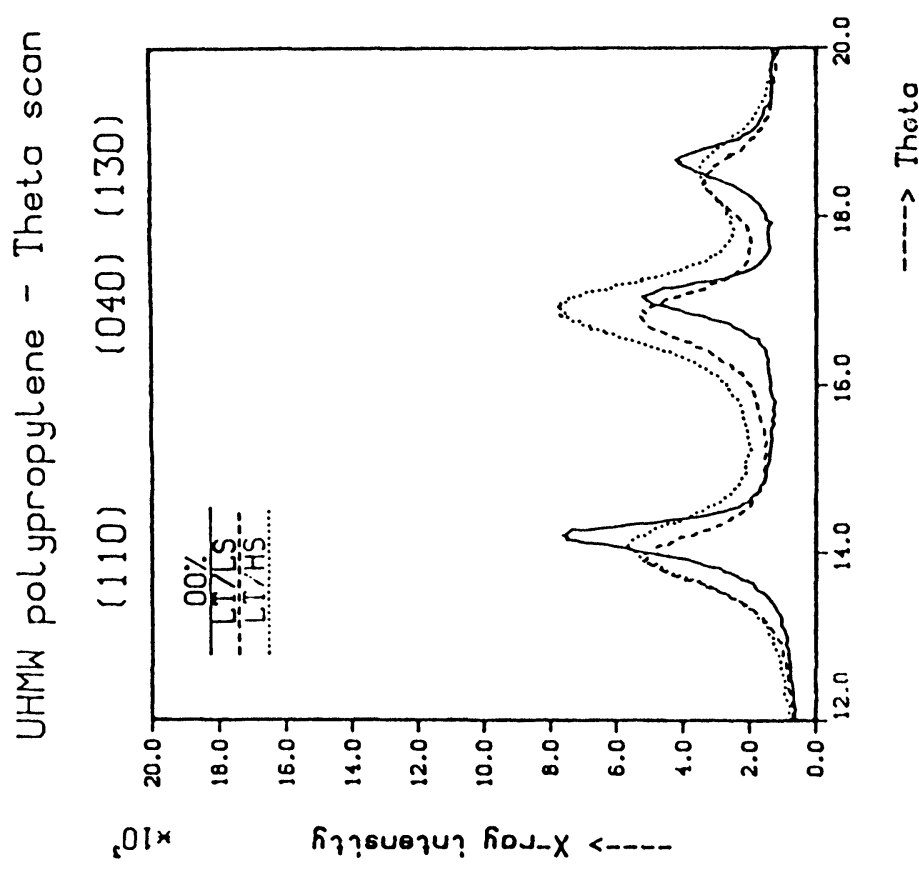

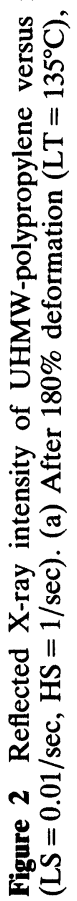


Table 1 (a) $\theta$ scan $(110) /(040) /\{130\}$ peak position $\left[^{\circ}\right]$ [deviation from ref. $\Rightarrow$ mean value]

\begin{tabular}{|c|c|c|}
\hline$M M W-P P:$ & $L T\left(135^{\circ} \mathrm{C}\right)$ & $H T\left(155^{\circ} \mathrm{C}\right)$ \\
\hline $\begin{array}{l}00 \text { (reference) } \\
\text { LS }(0.1 / \mathrm{sec}) \\
\text { HS }(10 / \mathrm{sec})\end{array}$ & $\begin{array}{l}14.19^{\circ} / 16.97^{\circ} / 18.64^{\circ} \\
14.00^{\circ} / 16.83^{\circ} / 18.45^{\circ} \\
\left.-0.19^{\circ} /-0.14^{\circ} /-0.19^{\circ} \Rightarrow-0.17^{\circ}\right) \\
13.75^{\circ} / 16.55^{\circ} / 18.20^{\circ} \\
\left(-0.44^{\circ} /-0.42^{\circ} /-0.44^{\circ} \Rightarrow-0.43^{\circ}\right)\end{array}$ & $\begin{array}{l}14.07^{\circ} / 16.91^{\circ} / 18.52^{\circ} \\
14.17^{\circ} / 17.03^{\circ} / 18.63^{\circ} \\
\left.+0.10^{\circ} /+0.21^{\circ} /+0.09^{\circ} \Rightarrow+0.11^{\circ}\right) \\
13.97^{\circ} / 16.83^{\circ} / 18.47^{\circ} \\
\left(-0.10^{\circ} /-0.08^{\circ} /-0.05^{\circ} \Rightarrow-0.08^{\circ}\right)\end{array}$ \\
\hline$U H M W-P P$ & $H T\left(135^{\circ} \mathrm{C}\right)$ & $L T\left(155^{\circ} \mathrm{C}\right)$ \\
\hline 00 (reference) & \multicolumn{2}{|c|}{ untreated $14.20^{\circ} / 17.02^{\circ} / 18.64^{\circ}$} \\
\hline LS-60 & - & $\begin{array}{l}14.11^{\circ} / 16.99^{\circ} / 18.53^{\circ} \\
\left(-0.09^{\circ} /-0.03^{\circ} /-0.11^{\circ} \Rightarrow-0.08^{\circ}\right)\end{array}$ \\
\hline LS-120 & ( 50 & $\begin{array}{l}14.06^{\circ} / 16.96^{\circ} / 18.54^{\circ} \\
\left(-0.14^{\circ} /-0.06^{\circ} /-0.10^{\circ} \Rightarrow-0.10^{\circ}\right)\end{array}$ \\
\hline LS-180 & $\begin{array}{l}13.92^{\circ} / 16.80^{\circ} / 18.39^{\circ} \\
\left(-0.28^{\circ} /-0.22^{\circ} /-0.25 \Rightarrow-0.25^{\circ}\right)\end{array}$ & $\begin{array}{l}14.18^{\circ} / 17.05^{\circ} / 18.65^{\circ} \\
\left(-0.02^{\circ} /+0.03^{\circ} /+0.01^{\circ} \Rightarrow+0.01^{\circ}\right)\end{array}$ \\
\hline HS-180 & $\begin{array}{l}14.10^{\circ} / 16.91^{\circ} / 18.5^{\circ} \\
\left(-0.10^{\circ} /-0.11^{\circ} /-0.14^{\circ} \Rightarrow-0.12^{\circ}\right)\end{array}$ & $\begin{array}{l}14.25^{\circ} / 17.11^{\circ} / 18.70^{\circ} \\
\left(+0.05^{\circ} /+0.09^{\circ} / 0.06^{\circ} \Rightarrow+0.07^{\circ}\right)\end{array}$ \\
\hline
\end{tabular}

(b) $\theta$ scan $110 / 040 / 130$ peak maxima $I\left[\right.$ ratio $\left.I_{i} / I_{00}\right]$

\begin{tabular}{lll}
\hline$M M W-P P:$ & $L T\left(135^{\circ} \mathrm{C}\right)$ & $H T\left(155^{\circ} \mathrm{C}\right)$ \\
\hline 00 (reference) & $6985 / 5258 / 3901$ & $6181 / 4684 / 3982$ \\
LS & $13125 / 16485 / 4607$ & $9544 / 26658 / 7017$ \\
& $(1.88 / 2.81 / 1.18)$ & $(1.54 / 5.69 / 0.76)$ \\
HS & $3801 / 6152 / 2006$ & $7395 / 10968 / 4499$ \\
& $(0.54 / 1.05 / 0.51)$ & $(0.84 / 2.34 / 1.13)$ \\
\hline$U H M W-P P:$ & $L T\left(135^{\circ} \mathrm{C}\right)$ & $H T\left(155^{\circ} \mathrm{C}\right)$ \\
\hline 00 (reference) & & untreated $7656 / 5274 / 4248$ \\
LS 60 & - & $4269 / 7492 / 3472$ \\
& & $(0.56 / 1.42 / 0.82)$ \\
LS 120 & - & $4920 / 11153 / 4483$ \\
& & $(0.64 / 2.11 / 1.06)$ \\
LS 180 & $5293 / 5301 / 3461$ & $7580 / 19041 / 6456$ \\
HS-180 & $(0.69 / 1.01 / 0.81)$ & $(0.99 / 3.61 / 1.52)$ \\
& $5755 / 7816 / 3595$ & $4793 / 8727 / 3962$ \\
& $(0.75 / 1.48 / 0.85)$ & $(0.63 / 1.65 / 0.93)$ \\
\hline
\end{tabular}

values for their deviation from the undeformed reference sample are listed for the different conditions examined. The peaks of the MMW-PP samples were measured from unrelaxed samples and therefore show higher shifts (up to $\sim 0.4^{\circ}$ ) than those from the UHMW-PP samples which were relaxed after deformation. Table $1 \mathrm{~b}$ gives the corresponding absolute and relative peak heights.

For a complete evaluation of the texture, it is necessary to measure the reflected intensity over the whole range of sample orientations. The results of these types of measurements are usually presented in form of stereographic projections, i.e., as pole figures. Some examples are given in Figure 3. The pole figure data are corrected and normalized to random intensity using the reference samples and the overall measured pole figure intensity. Due to the rotational 


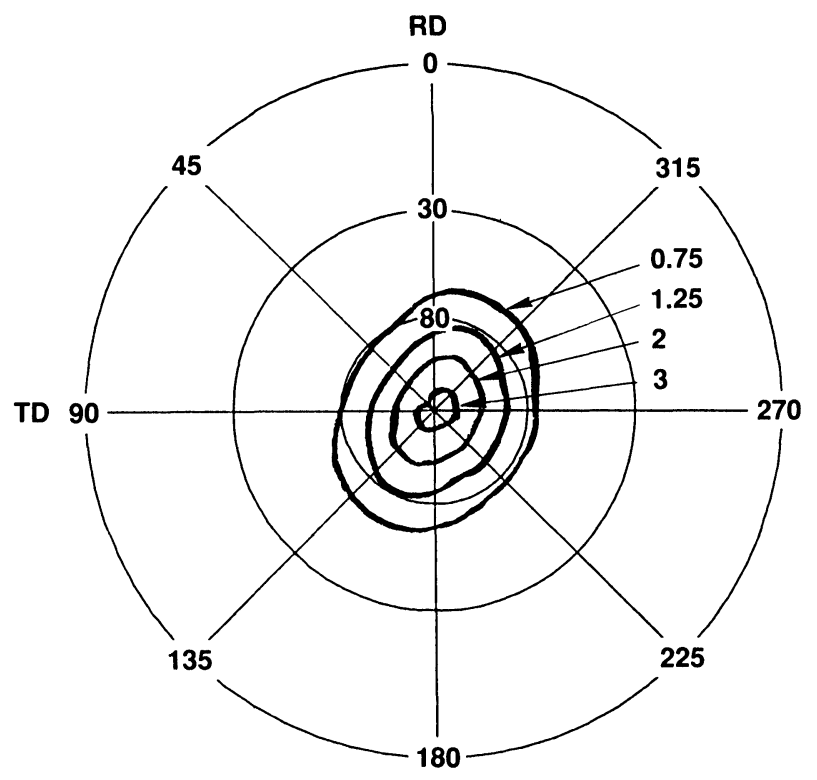

a) HT-LS

(040)

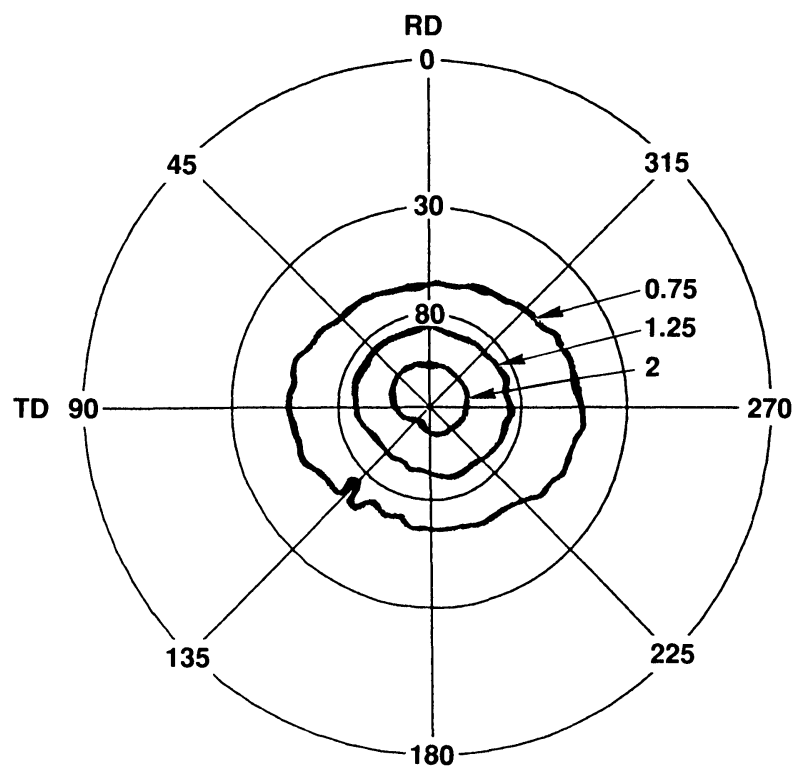

b) HT-HS

Figures 3a and 3b (040) pole figures of MMW-polypropylene (contour levels: 0.75-1.25-2-3) deformed at high temperature $=155^{\circ} \mathrm{C}(\mathrm{HT})$, at low strain rate $=0.1 / \mathrm{sec}$ (LS) and high strain rate $=10 / \sec (\mathrm{HS})$. 


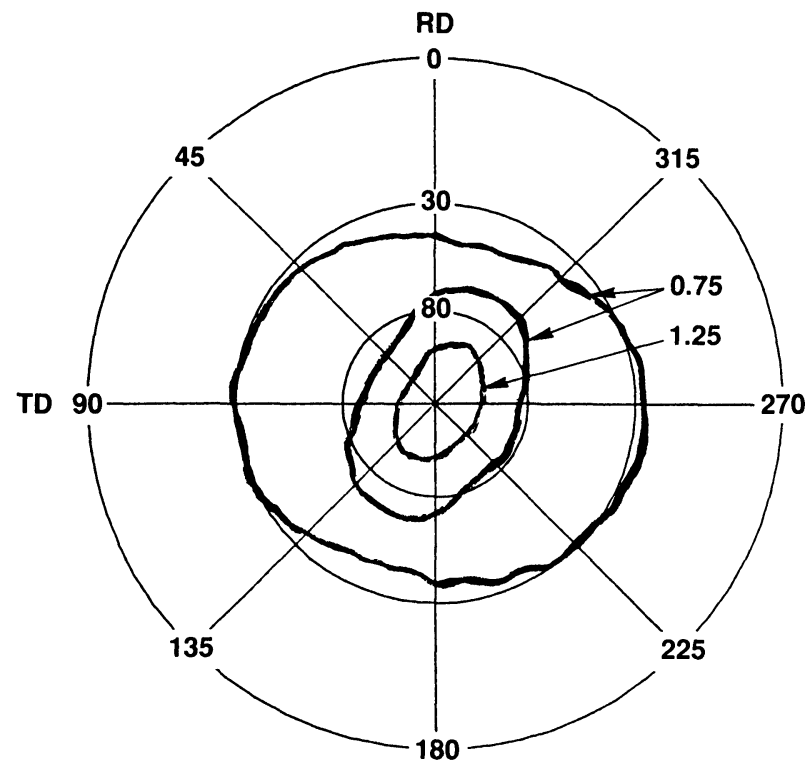

c) HT-LS

(110)

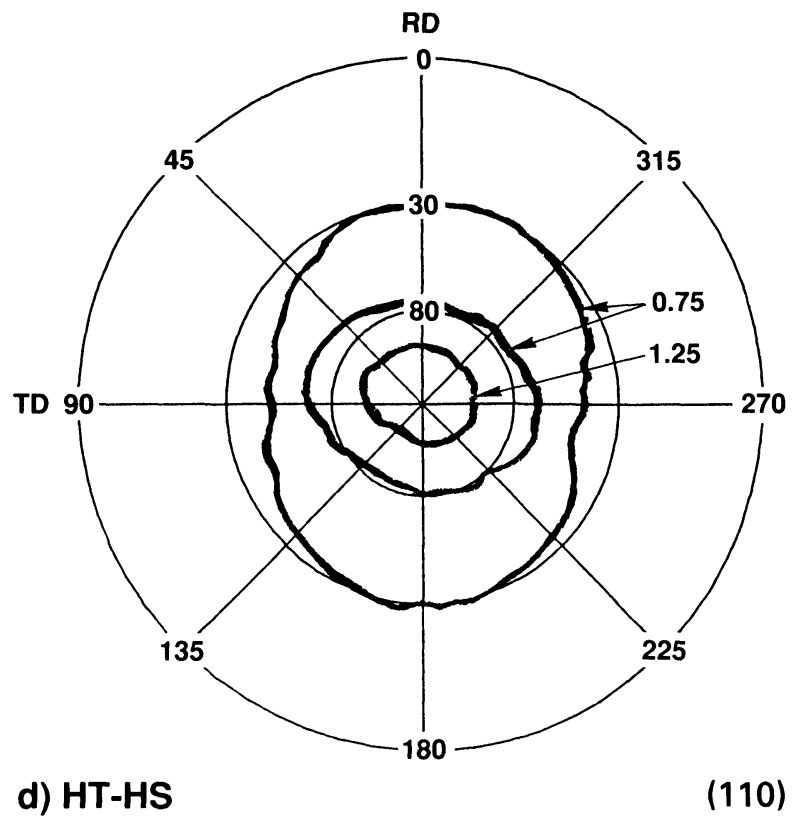

Figures 3c and 3d (110) pole figures of MMW-polypropylene (contour levels: 0.75-1.25-2-3) deformed at high temperature $=155^{\circ} \mathrm{C}(\mathrm{HT})$, at low strain rate $=0.1 / \mathrm{sec}$ (LS) and high strain rate $=10 / \sec (\mathrm{HS})$. 
symmetry of the uniaxial stress compression test, strong fiber textures occur. Therefore, it is possible to integrate the measured pole figure intensities for each pole circle $\left(\alpha=0^{\circ}\right.$ to $\left.90^{\circ}\right)$. These mean values of pole circle intensities are plotted versus $\alpha$ as in Figure 4 for the different strains in the UHMW samples. In Figures $5-8$, the relative differences to the reference sample [normalized with respect to the overall intensities, according to the method of Hirsch and Lücke, (1988)] are plotted which reveal the pure texture changes. This type of plot shows the relative variation in texture normalized with respect to the undeformed reference sample (intensity $=$ const. $=1$ ) and errors in absolute intensity levels are eliminated.

The evolution of texture during HT deformation shows the systematic (040) intensity increase with strain in the central position at $\alpha=0^{\circ}$ and a general decrease for $\alpha \geq 25^{\circ}$ can be noticed in Figures 4, 5 and 7. In all tests, the strongest effect can be observed in the HT/LS samples whose central (040) intensity increases significantly compared to the undeformed sample intensity (Figure 4). After normalization (Figures $5 \mathrm{~b}$ and $7 \mathrm{~b}$ ) a factor of $f \approx 2.3$ times the intensity of the undeformed (reference) sample is determined. For high strain rates $(\mathrm{HT} / \mathrm{HS})$ for both materials a significantly smaller texture effect $(f \approx 1.7)$ is found. The same is true for the samples deformed at the lower temperatures (Figures 5a and 7a). Here the UHMW samples show a somewhat weaker texture effect than the MMW samples, but consistently in both materials at low

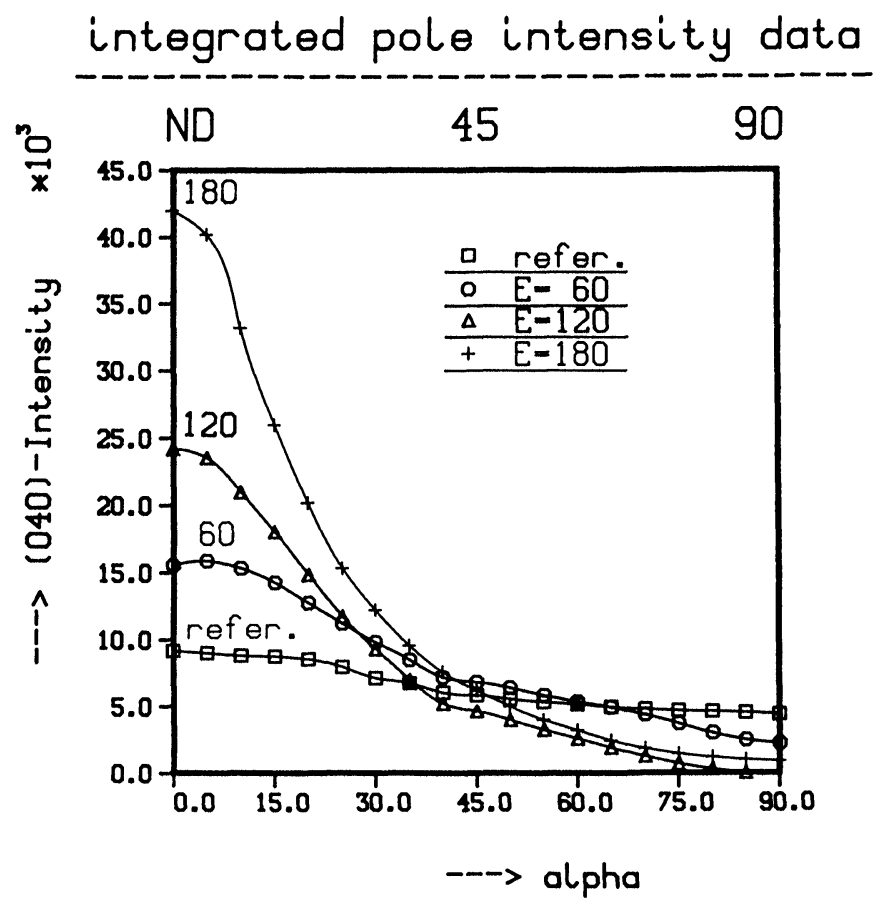

Figure 4 (040) mean pole intensities of UHMW-polypropylene deformed at $155^{\circ} \mathrm{C}(\mathrm{HT})$ at $\dot{\varepsilon}=1 / \mathrm{sec}$ (HS) to different strains $(0 \%, 60 \%, 120 \%, 180 \%$ thickness reduction). 


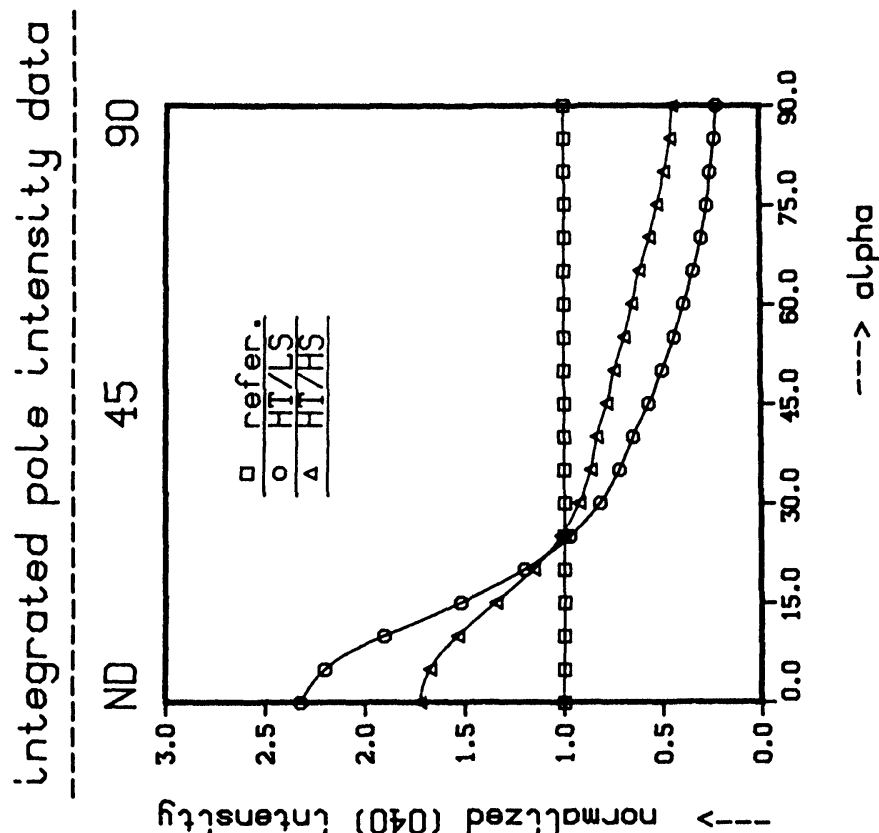

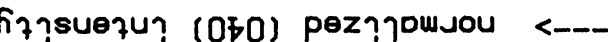

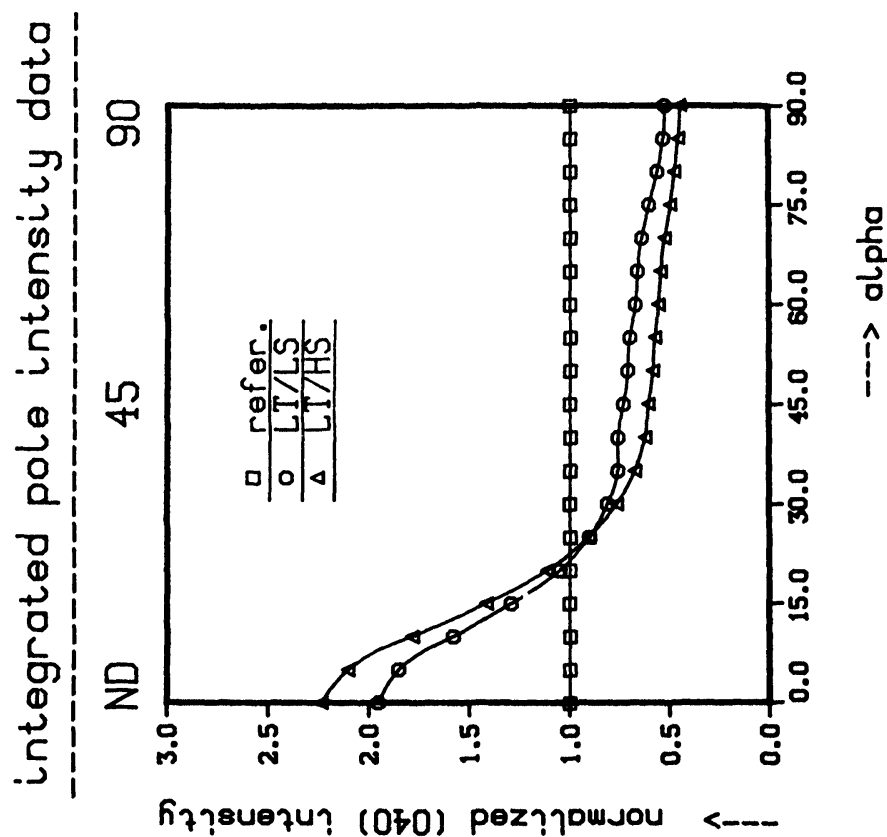

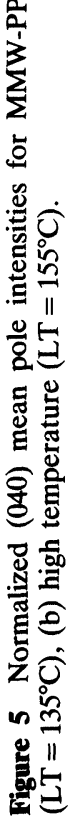




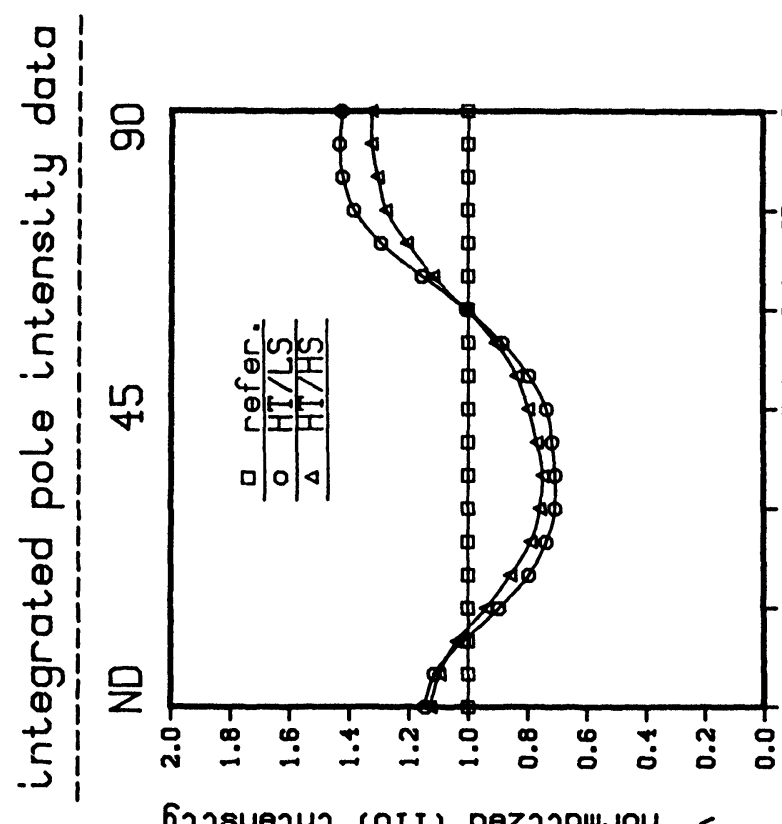



ศұ? suequ? (OII) pez?ךowsou <-

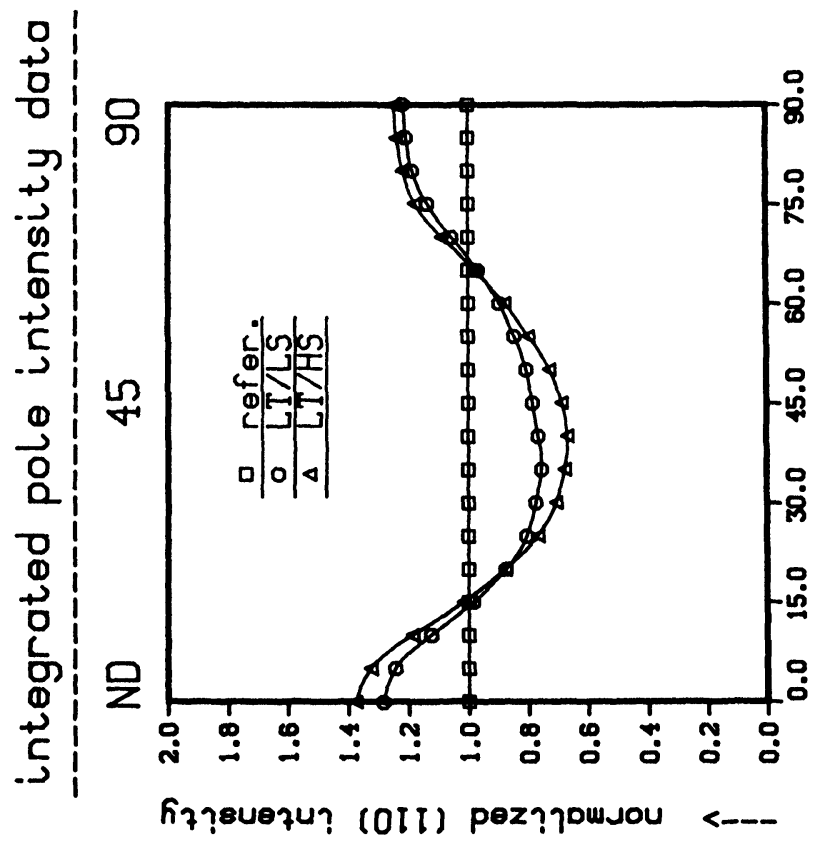

$\sum^{3}$

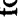

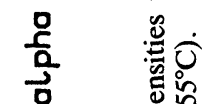

$8 \hat{\wedge} \quad . \triangleq$

- $\quad \hat{\text { i }}$

ङ

을 옳

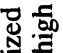

灵

z⿺辶

เ

紫 

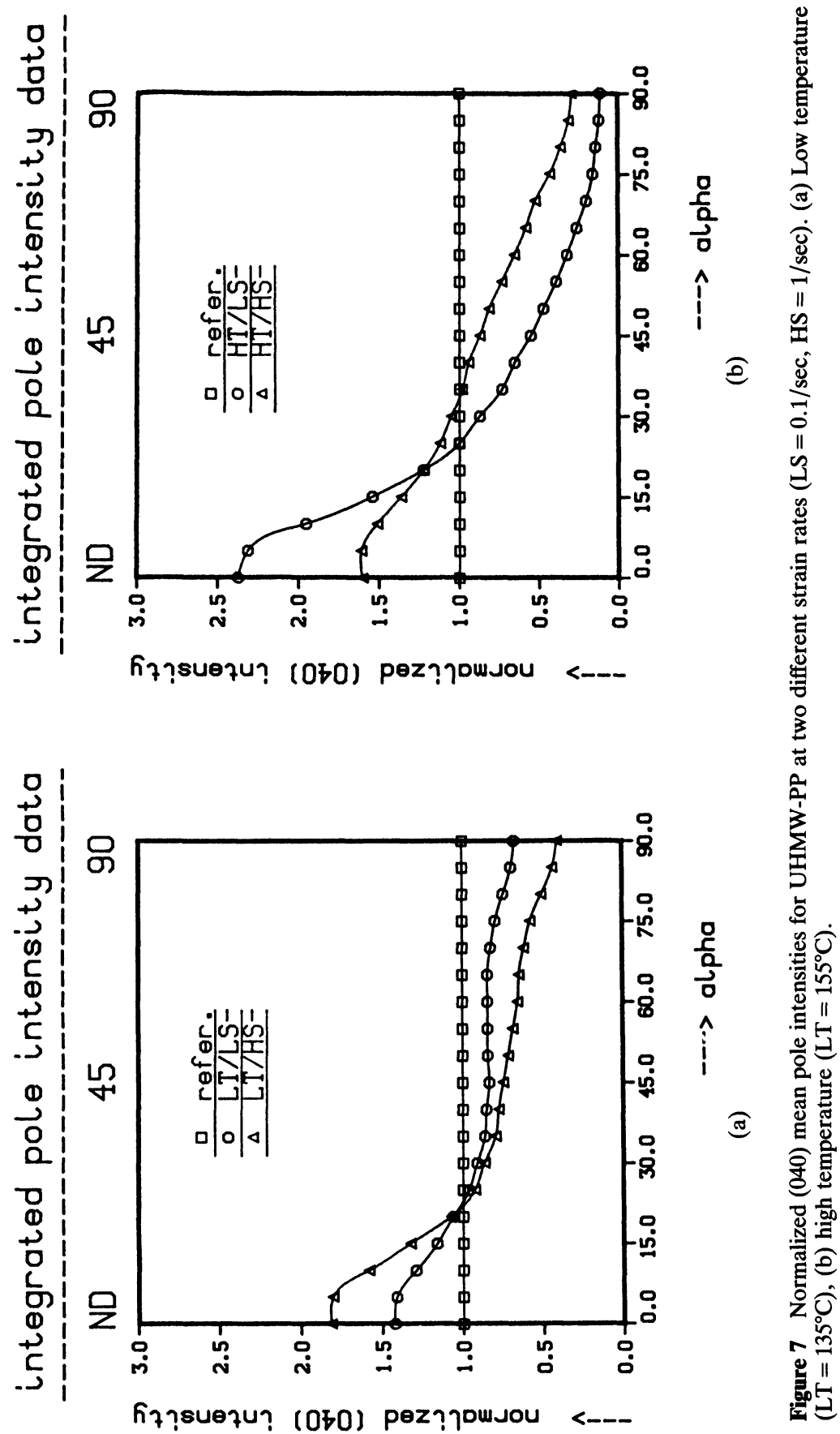

음 ब

o

i II

อิ

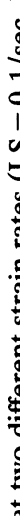




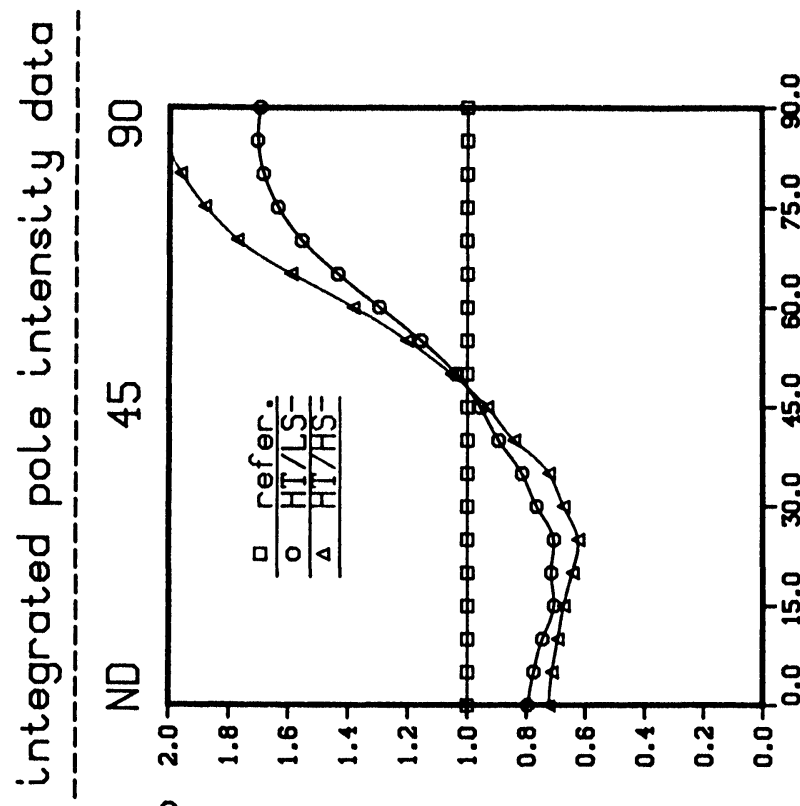

f7? suequ? (OII) pez?powsou

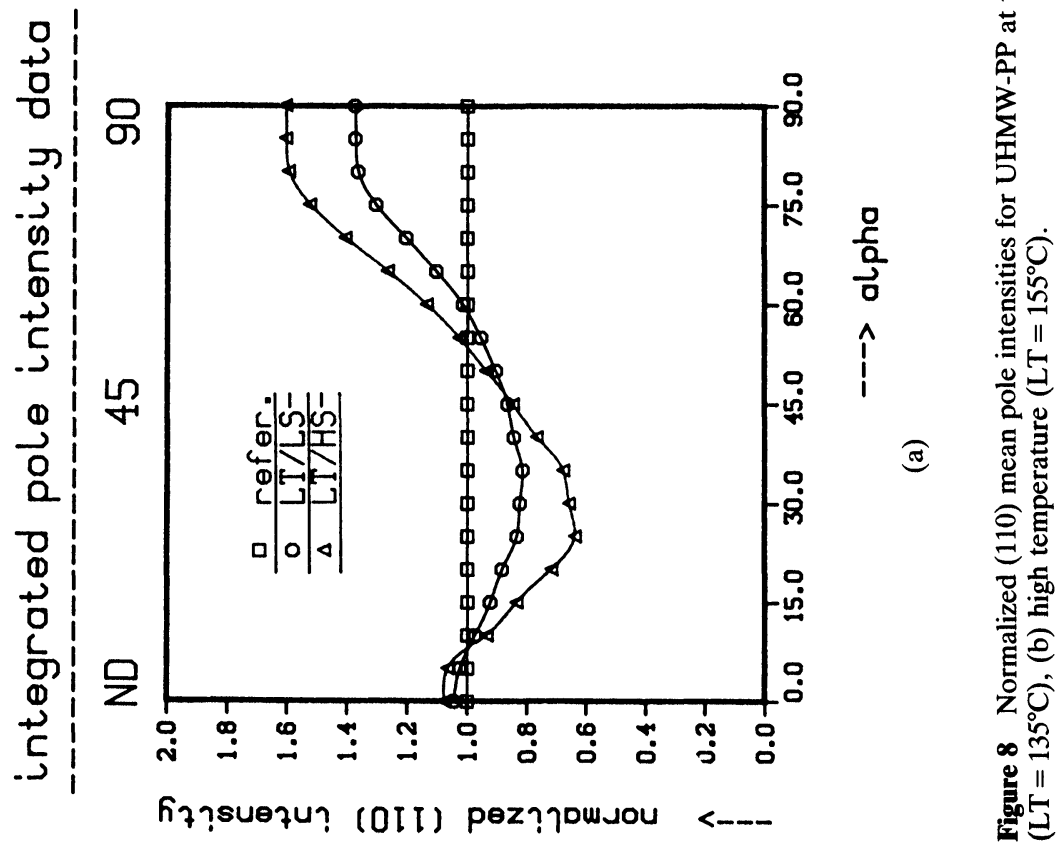


temperature, the (040) peak at low strain rate (LT/LS) is smaller than that at high strain rate (LT/HS).

For the (110) pole figures, intensity peaks occur at $\alpha=0^{\circ}$ (except for the HT-UHMW samples, Figure 8b), and in all materials, a broad maximum for (110) at $\alpha>70^{\circ}$ is found (Figures 6 and 8). The latter texture effect is most pronounced for the HT samples (Figures $6 \mathrm{~b}$ and $8 \mathrm{~b}$ ) while the former appears to be stronger for the LT samples (Figures 6a and 8a). In all samples, a broad decrease in intensity-with respect to the reference sample-occurs around $\alpha \approx 35^{\circ}$.

\section{Stress-Strain Behavior}

The evolution of stress was measured during compression. For subsequent relaxation tests some of the samples were kept at constant strain after $180 \%$ thickness reduction where the stress gradually drops due to viscous material flow. Figure 9 shows the results of the stress/strain measurements for the MMWpolypropylene samples. (For the UHMW-polypropylene, very similar curves were found.) One general observation is a significantly higher stress at high strain rate and at lower temperature. The absolute level of initial stress for sample HT/HS is similar as that for sample LT/LS (about $5 \mathrm{MPa}$ ). The strain hardening effect in the HT/HS stress/strain curve, however, is higher than that at low T. While both LT samples show similar strain hardening, this material parameter significantly differs for the HT samples (which agrees to the observed variation in textures). For the HT/LS sample, where strongest texture effects are found, the strain hardening is lowest and vice versa for the HT/HS data.

The stress relaxation curves after $180 \%$ strain are plotted in Figure 10. Holding the samples at constant strain $(180 \%)$, the stress drops quickly from the maximum, lead $\sigma_{\max }$ and gradually approaches a constant level, i.e., the fully

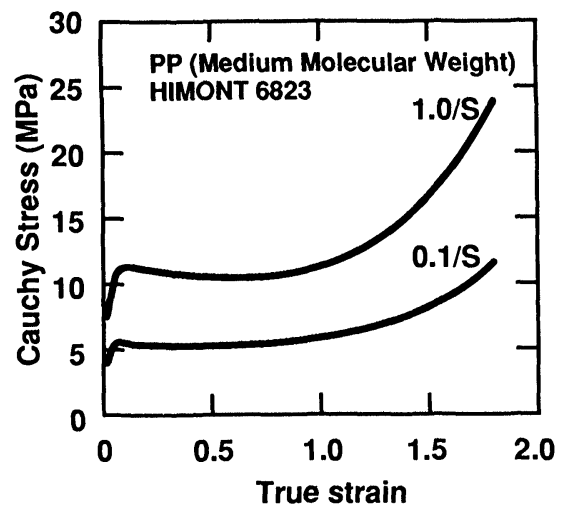

(a) Uniaxial compression $\left(135^{\circ} \mathrm{C}\right)$

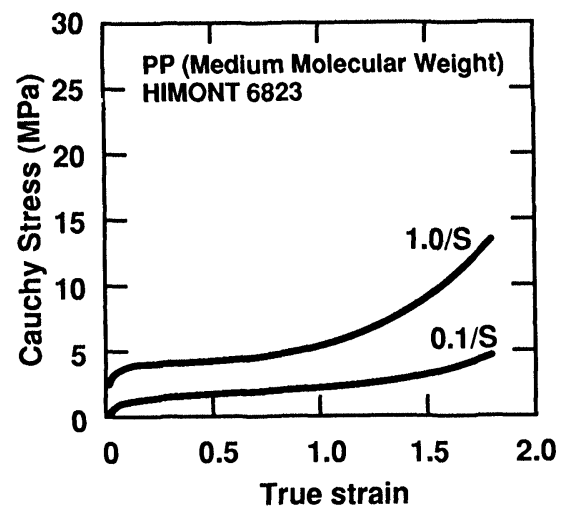

(b) Uniaxial compression $\left(155^{\circ} \mathrm{C}\right)$

Figure 9 Stress strain curves for uniaxial compression of MMW-polypropylene at different strain rates. (a) $135^{\circ} \mathrm{C}$, (b) $155^{\circ} \mathrm{C}$. 


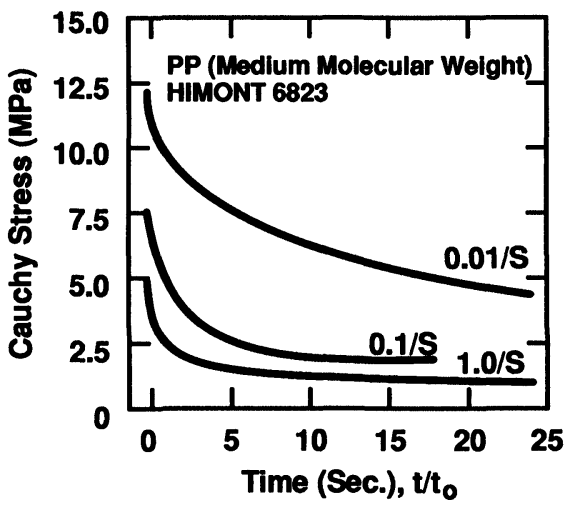

(a) Stress relaxation (135 ${ }^{\circ} \mathrm{C}, 180 \%$ strain)

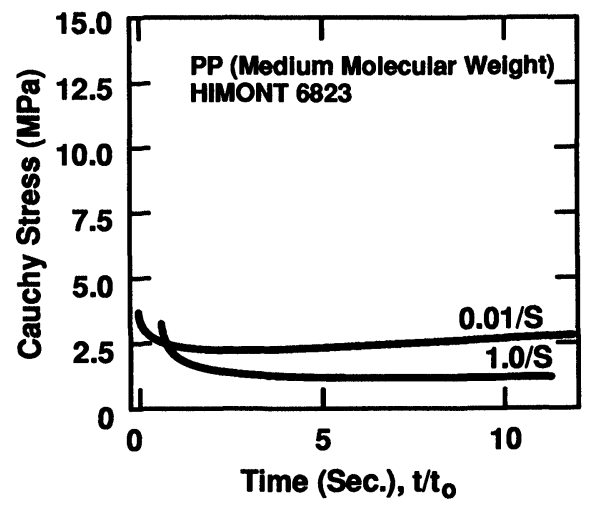

(b) Stress relaxation ( $155^{\circ} \mathrm{C}, 180 \%$ strain)

Figure 10 Stress relaxation curves for MMW-polypropylene after $180 \%$ compression at different strain rates. (a) $135^{\circ} \mathrm{C}$, (b) $155^{\circ} \mathrm{C}$.
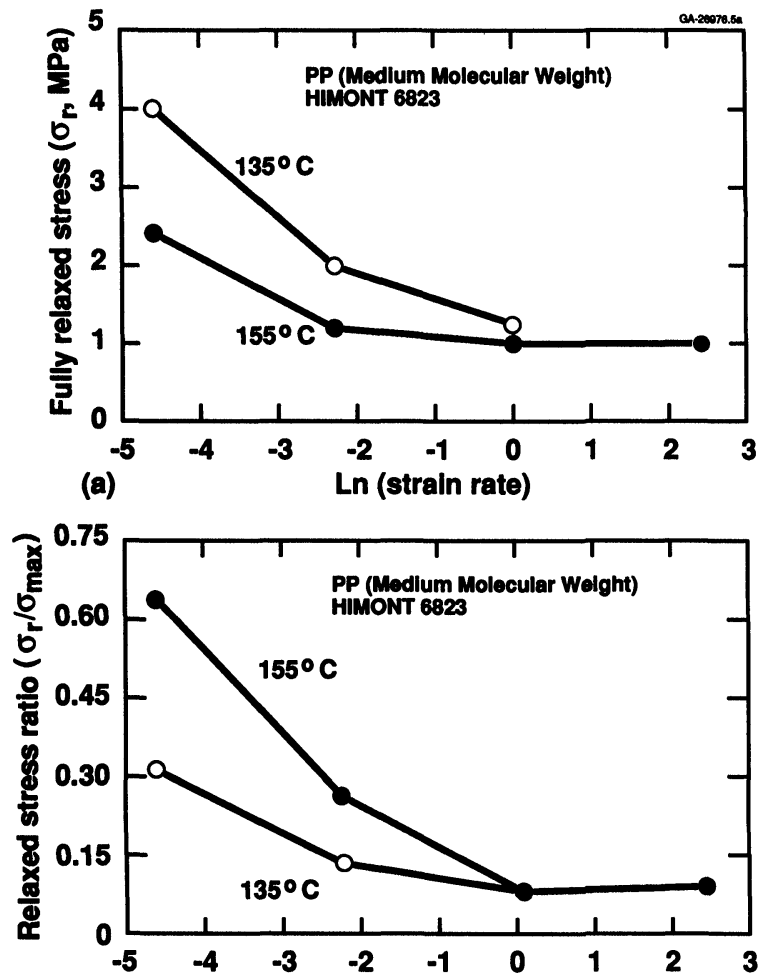

(b)

Ln (strain rate)

Figure 11 Relaxation stress data of MMW-polypropylene as function of strain rate. (a) Fully relaxed stress $\sigma_{r}$, (b) $\sigma_{r}$ normalized to $\sigma_{\max }$. 
relaxed stress, $\sigma_{r}$. It is interesting to note that the stress drop during relaxation is stronger for higher strain rates and reaches a lower level of the fully relaxed stress, even if the initial stress $\sigma_{\max }$ was higher. This effect is shown in Figure 11a where the full relaxation stresses $\sigma_{r}$ are plotted for the two deformation temperatures as function of logarithmic strain rate. The effect seems to be stronger for the low temperature $\left(135^{\circ} \mathrm{C}\right)$ deformed samples than for $155^{\circ} \mathrm{C}$ (Figure 11a), but this is reversed when $\sigma_{r}$ is normalized to $\sigma_{\max }$ (Figure 11b).

\section{DISCUSSION}

\section{Crystal Structure of Isotactic Polypropylene}

By annealing polypropylene in a temperature range between $100^{\circ} \mathrm{C}$ and $155^{\circ} \mathrm{C}$, thermal induced crystallization occurs. Spherulitic grains grow and form crystal lamellae in radial direction. In the present material in MMW, $\sim 62 \%$ and UHMW $\sim 65 \%$ crystallinity was produced. The crystal structure of isotactic polypropylene is built up by the systematic arrangement of the helical (worm-like) molecules in a folded structure bounded by van der Walls forces (schematically shown in Figure 12). The long (worm) axis then forms the $c$-axis (=helix or molecular axis), which

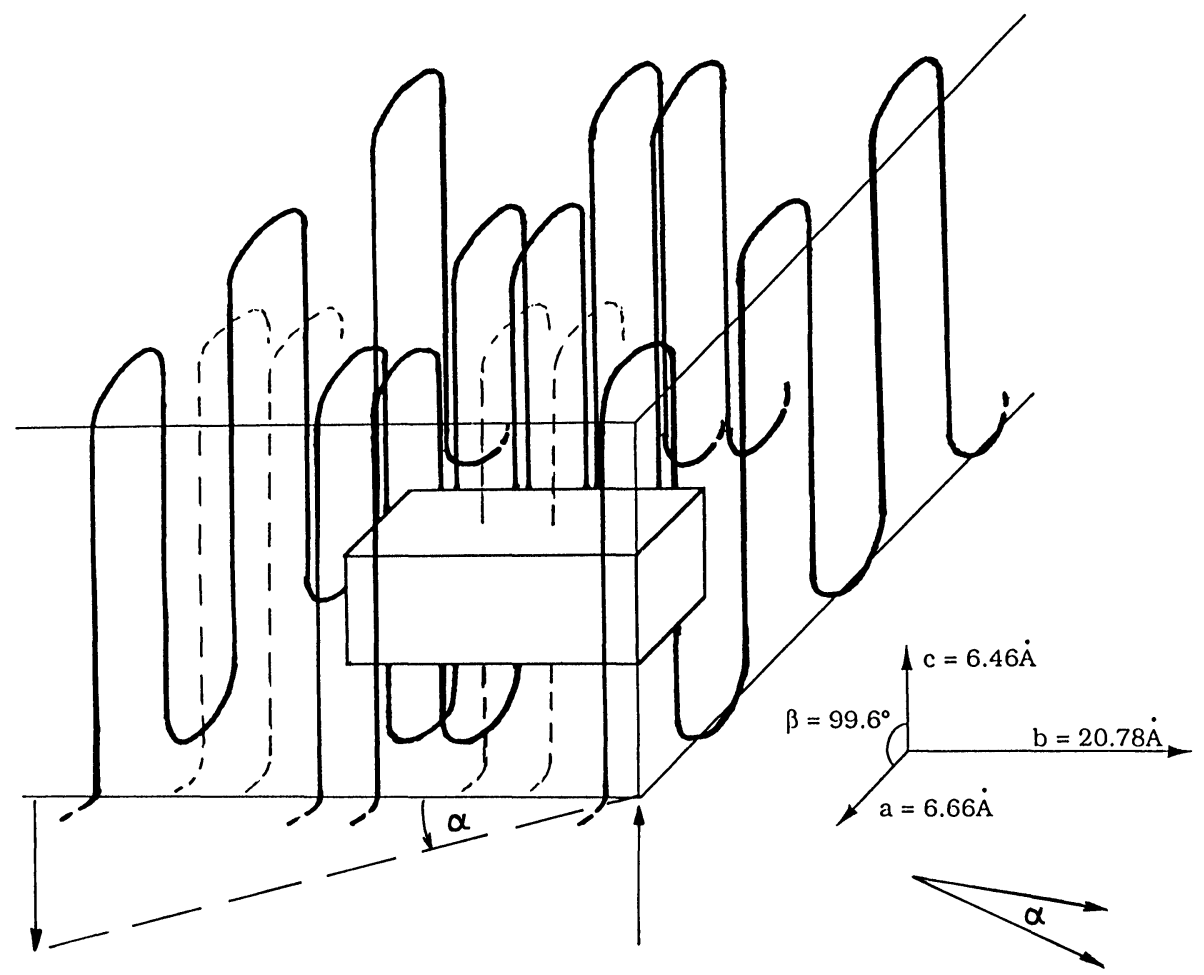

Figure 12 Schematic plot of the helical molecular structure of crystalline isotactic polypropylene (for better clarity, some molecular chains are omitted). The arrows indicate the shear direction by planar glide on crystalline planes normal to the $b$-axis and $\alpha$ gives the angle of reorientation of a direction defined in the sample coordinates (e.g., compression axis). 
becomes unfolded and realigned during plastic deformation. The direction of the worm-like arrangement of the molecules is the $a$-axis while the plane where the molecules are arranged beside each other is normal to the $b$-axis of the crystal unit cell. The latter can directly be measured in X-ray diffraction as the (040) reflex. The (110) reflex (i.e., the normal of the (110) plane) is closer to the $a$-direction, forming a $72.5^{\circ}$ to the $b$-axis.

During plastic deformation the molecules shear in direction of the $c$-axis and so rearrange. In a tensile test this rotates the unit cell until the $c$-axis is aligned parallel to the tensile axis (Samuels, 1974). In a uniaxial stress compression test, the rotation will align the $c$-axis normal to the compression direction. Mainly those crystalline fibers will deform by shear which are located in the spherulites under a certain angle to the principal strain directions. After their rearrangement which is accompanied by low strain hardening and the alignment of lamellae normal to the direction of stress is complete, plastic deformation in this mode is exhausted which leads to a stress increase and to finally fracture.

\section{Texture Evolution}

The observed texture effects due to compression at elevated temperatures clearly reveal the rotation of the $b$-axis of the crystalline structure normal to the compression axis. This is indicated by the peak increases of the $(040)$ pole figure center $\left(\alpha=0^{\circ}\right)$ and of the (110) poles at $\alpha \gtrsim 70^{\circ}$. The rotation of the $b$-axis normal to the compression axis-where the $c$-axis lies in the compression plane-can be achieved by a deformation mechanism in which glide occurs on planes normal to the $b$-axis, i.e., on the planes formed by the folded molecules plotted in Figure 12. Since in tensile deformation, the $c$-axis is indicated as the major slip direction-as here its alignment with the tensile axis causes the observed texture effect (Samuels, 1974) - this reveals the complete mechanism of deformation of crystalline isotactic polypropylene as shear of the worm-like molecules on the $b$-plane parallel to the $c$-axis. This also means that the structural changes during compression deformation are widely preserving the worm-like molecular arrangement as shearing occurs parallel to the $b$-plane and the lamellae are only displaced with respect to each other and the chains are not distorted. This and a possible rearrangement of the molecules at the high temperature explains why the molecular (crystalline) structure is still present after high strain. As the texture evolution was strongest for the high temperature/low strain rate (HT/LS) deformed samples under these conditions, the crystalline part of the structure participates strongest in the overall deformation by this deformation mode.

The deformation geometry is different at low temperature as here an additional central (110) peak is found experimentally (Figures 6a, 8a). This means that at low temperature, the preference of the $b$ plane as major slip plane is lost which indicates a strong temperature dependence of the necessary shear stress. However, still the alignment of the $c$-axis normal to the compression direction occurs but without any preferred shear plane. Also, at higher strain rate (HT/HS) this mechanism of planar slip is much less effective as the texture evolution is significantly reduced. This observation supports the interpretation that the molecular binding forces between the $b$-planes (Figure 12) are strengthening and 
lamellar mobility decreases with temperature [as long as the processing temperature is ranged above the crystallization temperature (about $100^{\circ} \mathrm{C}$ ) and below the melting point (about $165^{\circ} \mathrm{C}$ )]. At high strain rate the material shows less crystalline orientation change and an increased contribution of the amorphous volume to the overall deformation must take place.

With a lower activation energy for plastic flow the amorphous part contributes more deformation at low temperature and high strain rate. The strong peak shifts of the X-ray reflexes (especially in the quenched MMW-PP samples) can be explained that way as they reveal strong residual stresses probably caused by the viscoelastic nature of the amorphous volume. These effects are most pronounced in the LT/HS material (Table 1a), where also the central (110) peak is strongest (Figures 6-8a). As the crystalline volume is always surrounded by amorphous structures, changes in constraints must be considered and the amorphous structure might impose higher reaction stresses at low temperature and high strain rates. The crystalline volume itself does not necessarily need to participate in plastic deformation as a simple rigid body rotation effect could produce this texture effect of a purely aligned $c$-axis. There the crystalline fibers-embedded in the amorphous volume-are rotated parallel to the compression plane by deformation of the surrounding matrix.

\section{Stress/Strain Behavior}

The observed texture evolution during deformation has been correlated with the characteristic stress strain behavior in polypropylene (Samuels, 1974). As long as crystalline volume is oriented such that the shear direction or the glide plane forms a certain angle $\left(0^{\circ}<\alpha<90^{\circ}\right)$ with the tensile (or compression) direction, deformation is relatively easy and the flow stress remains relatively low. But as more planes are rotated parallel to the tensile axis (or normal to the compression direction) by the slip process-as indicated by the texture effects observed-their orientation-and so the texture-gets progressively more unfavorable for this kind of slip and so the stress necessary to maintain deformation must increase. This effect can explain the characteristic stress strain curves of this material (Figure 9) where the final increase in stress occurs when the alignment is almost complete. Then the crystallites no longer participate in deformation and other deformation modes become active which may affect the crystallinity of this structure (crystal cleavage). Finally fracture occurs where the type of texture may also play an important role as cracking in the $b$-axis direction implies cleavage between the molecule lamellae (Samuels, 1974).

However, the contribution of the crystalline phase above does not explain all the effects observed, as the final stage of strain hardening is more pronounced for the high strain rate where the evolution of texture was less pronounced. Here the interaction of crystalline and amorphous phases must be considered. Semicrystalline polypropylene is composed of amorphous and crystalline phases in a very entangled fashion. Based on monotonic compression and stress relaxation data (discussed below), it is inferred that at high strain rate, due to its viscoelastic nature, the deformation of the amorphous phase which contributes to the increase in reversible energy is more pronounced than that of the crystalline phase. At the later stage of deformation, this amorphous contribution can lead to extensive hardening in the stress-strain curve. At lower deformation rate, the 
crystalline volume contributes more deformation (which is irreversible) than the amorphous part. This is confirmed by the observed material directionality (or texture intensity of the key structural direction) which increases with low deformation rate.

Typical flow stress measurements for MMW-PP at different strain rates and temperatures (Figure 9), show a systematic increase of flow stress with the increase of strain rate at the monotonic loading situation. The fully relaxed stress decreases with the increase of strain rate (Figure 10) which is illustrated in Figure 11a where a full set of this fully relaxed stress with respect to temperature and strain rate is plotted. This is particularly true for high temperature (HT) case and can be illustrated in Figure 11b, in which the fully relaxed stress $\left(\sigma_{r}\right)$ is normalized with the maximum compressive stress $\left(\sigma_{m}\right)$ prior to the relaxation as $\sigma_{r} / \sigma_{m}$. This normalized stress ratio indicates the approximate ratio of irreversible energy with respect to the total energy at various strain rates and temperatures since the strain is kept constant. Obviously, there is more conversion to nonviscous energy at high temperature (HT) than at low temperature (LT), as well as for lower strain rate (LS) than for higher strain rate (HS). The majority of viscous energy (stored in the amorphous volume) is damped out at the beginning stage of relaxation. The remaining nonviscous energy which corresponds to the relaxed stress at a constant strain can be related to the texture development of the crystalline part as more texture is developed with a higher level of irreversible energy. Hence, the slower the deformation process is, the stronger the texture strength and the more nonviscous energy is stored.

\section{Modeling of Stress/Strain Effect}

The overall perspective on orientation for semicrystalline polymer, which includes amorphous orientation and crystalline texture, can be rationalized as follows: By assuming a two-phase system, the orientation development as well as structure evolution and the property outcome for semicrystalline should be contributed by both phases, amorphous and crystalline. Since the summation of the amorphous orientation and the crystalline texture contributes to the total orientation, we need to evaluate the orientation on both phases individually and then combine them to conclude on the overall orientation change with deformation. The crystalline texture development as discussed in the previous section indicates a significant increase with the decrease of strain rate at high temperature (HT). It is less significant at low temperature (LT) and there seems to be less sensitive to strain rate. The former implies an easy glide mechanism with a preferred slip plane (where the surrounding amorphous part may well get into a viscous flow) while the latter is more a rigid body rotation of the crystalline fiber dominated by the deformation of amorphous phase. Although there is no amorphous orientation information at this time, it is speculated that there is less amorphous chain orientation in HT due to the viscous flow and more amorphous contribution to the overall orientation at LT because of the increase of chain mobility above $100^{\circ} \mathrm{C}$.

In the following, a simple analogy is proposed to explain the combined contribution of flow stress from the amorphous and crystalline phases. Assuming the free energy and strain of this two-phase system $\Psi, E$, are additive (affine) by 
both phases, thus

$$
\begin{gathered}
\Psi=\Psi_{a},\left(E_{a}, q_{a}, T\right)+\Psi_{c}\left(E_{c}, q_{c}, T\right) \\
E=(1-\beta) E_{a}+\beta E_{c}
\end{gathered}
$$

where the subscripts of $a$ and $c$ represent tor amorphous and crystalline phases, $E_{i}$ and $q_{i}$ are strain tensor (Lagrangian or Update-Lagrangian) and internal state variable tensor, respectively, for both phases, $\beta$ is the volume fraction of crystallinity and $T$ is the absolute temperature. Then, the stress state, $\sigma$, determined by the recoverable energy, can be summed linearly by

$$
\sigma=\left.\frac{\partial \Psi}{\partial E}\right|_{q}=(1-\beta) \sigma_{a}+\beta \sigma_{c}
$$

where $\sigma_{a}$, the amorphous part of stress state, is viscoelastic in nature, and $\sigma_{c}$, the crystalline part of stress state, is viscoplastic in nature. The evolution equation for each phase is a functional form of the free energy gradient as:

$$
\begin{aligned}
& \stackrel{\nabla}{q}_{a}=F_{a}\left(\frac{\partial \Psi_{a}}{\partial q_{a}}\right) \\
& \stackrel{\nabla}{q}_{c}=F_{c}\left(\frac{\partial \Psi_{c}}{\partial q_{c}}\right)
\end{aligned}
$$

For large deformation, Jaumann rate is adopted for evolution of internal state variable. It implies that the orientation of the amorphous part is related with the rotation of the chain configuration, and the texture of the crystalline part is relevant to the rotation of the lattice. Note that the interaction between these two phases could be described by the evolution equation by introducing a coupled term of $q_{i}$ in the free energy formation. A detailed functional form will be discussed elsewhere (Chen-Tsai et al., 1990), the principles of strength evolution with orientation of both amorphous and crystalline phases can be described as follows:

The resulting flow stress development for high and low strain rate cases at monotonic loading condition may be illustrated in the form shown in Figures 13

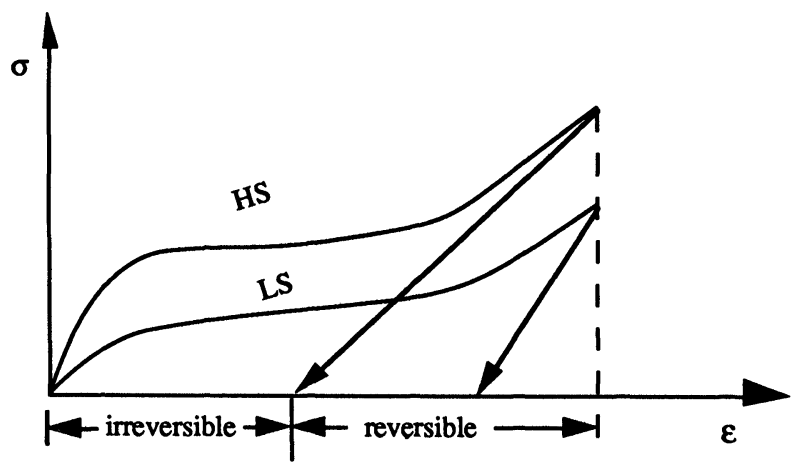

Figure 13 Schematic plot of the contributions of reversible (viscoplastic) and irreversible (crystallineplastic) strain to plastic deformation at different strain rates. 


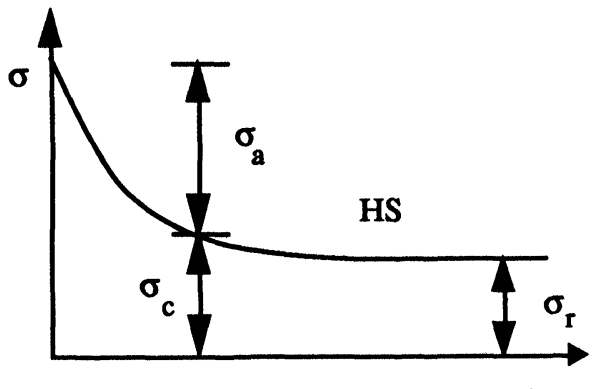

(a)

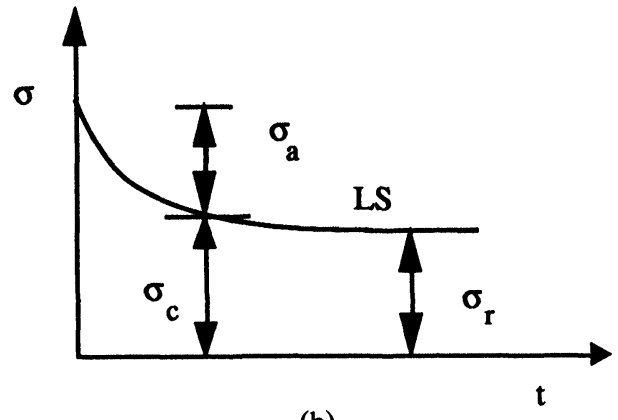

(b)

Figure 14 Schematic plot of stress relaxation curves for different strain rates. (a) High strain rate (HS), (b) low strain rate (LS).

and 14, where the unloading curves (Figure 13) are speculated based on stress relaxation data (schematically plotted in Figure 14). Note that even at zero stress after unloading, the deformation may be continuously recovered, depending on the degree of contribution from the amorphous part. For the high temperature case, not much chain configuration changes in the amorphous volume due to viscous flow (but viscous flow is still rate dependent). Thus, the deformation at lower strain rate (LS) contributes more to irreversible deformation than that at higher strain rate (HS). During relaxation, the majority of the viscous part will be diminished instantaneously and the remaining part will be decayed until the fully relaxed stage is reached. So a higher strain rate (HS) will lead to a higher stress drop at the beginning stage of relaxation due to more amorphous volume contribution while a lower strain rate (LS) shows a lower drop as it contains more irreversible deformation, stored in the crystalline volume (which is correlated with the texture).

For the low temperature case, the change of the amorphous chain configurations become more important. Hence, during unloading to zero stress, these may still have more strain reaction with time. It results in amorphous reorientation but contributes less to the crystalline texture evolution.

\section{SUMMARY}

The observed texture effects can help to analyze the underlying mechanism of deformation in polypropylene and interpret the stress strain behavior found in these materials under different deformation conditions. The results might look confusing at first glance but are reproducible in both materials investigated and can be interpreted as the overlapping contribution of two different structures, namely the crystalline fibers and the surrounding amorphous volume. Both structural parts are contributing differently to deformation and stress strain behavior and their deformation processes must have different activation energies which can explain the opposite switching in amount of crystalline deformation with strain rate at low and high deformation temperature.

From the present study of texture and strength evolution in high temperature deformed polypropylene, the following observations and conclusions can be 
summarized:

- The type of texture evolution shows a major peak increase in the (040) and a minor one in (110) pole figure center which reflects the rotation of the long $c$-axis (backbone axis) normal to the compression axis.

- The dependence of texture on temperature and strain rate with the stronger formation of a central (040) texture peak indicates that at low strain rate and high temperature a more selective (planar) glide mechanism exists with a preferred glide plane normal to the crystallographic $b$-axis.

- The stress/strain curves show an easy glide range in the early stages of deformation which can be associated with texture evolution and amorphous reorientation effects.

- The degree of texture intensity as a function of deformation conditions shows that the crystalline volume contributes more to deformation at low strain rates and at high temperatures where strain hardening is least. Thus it can be concluded that the evolution of a crystallographic texture by planar slip contributes less to strain hardening than assumed previously.

- The X-ray peak shift shows that after quenching, residual stresses are present especially after low temperature and high strain rate deformation.

- The stress relaxation data show a higher stress drop after high strain rate deformation which can be interpreted as due to the viscoelastic nature of the amorphous phase. It contributes less to deformation at a lower absolute stress level and at low strain rate where more deformation can occur in the crystalline phase.

\section{Acknowledgements}

Establishing the deformation testing techniques by V. M. Sample and fruitful discussions with Charlotte Chen-Tsai, L. A. Lalli and Rolf Rolles are gratefully acknowledged. Special thanks to Patti Napolitano and Susan V. Guerrini for typing the manuscript and plotting some of the figures.

\section{References}

Austen, A. R. and Humphries, D. V., "Oriented Semi-Crystalline Polymer Product and Method and Apparatus for Producing Such Product," U.S. Pat. 4,282,277, 1981 August 04.

Barnes, J. D. and Clark, E. S., "An Automated Apparatus for X-ray Pole Figure Studies of Polymers," in "Computer Applications in the Polymer Laboratory," ed. T. Provder, American Chemical Society, pp. 140-154, 1986 May.

Chen-Tsai, C. H., Wang, P. T. and Hirsch, J. R., "Processing-Structure-Property Relationship in Continually Forged Semicrystalline Polymer Sheets," 33rd IUPAC International Symposium on Macromolecules, 1990 July $08-13$.

Hiltner, A., Tang, H. I., Pan, S. J. and Baer, E., "Biaxial Orientation of Polypropylene by Hydrostatic Extrusion," Proceeding of Orientation of Polymer at Solid State, Society of Plastic Engineers, pp. 60-70, 1987, Atlantic City, NJ.

Hirsch, J. R. and Lücke, K., "Description and Presentation Methods for Textures," Textures and Microstructure Vols. 8 and 9 (Wasserman Memorial Edition) (1988), p. 131-151.

Katti, S. S. and Schultz, J. M., "The Microstructure of Injection-Molded Semicrystalline Polymers: A Review," Polymer Engineering and Science, Vol. 22, No. 16, 1982 November, pp. 1001-1017.

Lo, Y. C. and Humphries, D. V., "Methods of Producing Biaxially-Oriented Polymer Product, and the Product Produced Thereby," U.S. Pat. 4,874657, 1989 October 17.

Osaki, S., "A New Method for Quick Determination of Molecular Orientation in Poly(ethylene 
terephthalate) Films by Use of Polarized Microwaves," Polymer Journal, Vol. 19, No. 7, pp. 821-828, 1987.

Di Pede, S. and Woodhams, R. T., "Deep Drawing Uniaxially Ordered Polypropylene Sheet," Proceedings of 47th Annual Technical Meeting, Society of Plastic Engineers, pp. 427-430, 1989 May, New York, NY.

Samuels, R. J., "Structured Polymer Properties," John Wiley \& Sons, New York, London, Sydney, Toronto, 1974.

Wang, P. T., Chen-Tsai, C. H. and Sample, V. M., "Biaxial Orientation of Polypropylene Under Solid State Compression," Society of Plastic Engineers, 47th Annual Technical Conference, 1989 May 01-04, New York, pp. 422-426. 